\title{
On a Wiener-Poisson equation with rapidly fluctuating coef- ficients: application to large deviations
}

\author{
Alioune Coulibalya,*, Mouhamad Mounirou Allaya ${ }^{\mathrm{b}}$ \\ ${ }^{a}$ Amadou Mahtar Mbow University of Dakar, Senegal. \\ ${ }^{b}$ Iba Der Thiam University of Thies, Senegal.
}

\begin{abstract}
In this paper, we deal with a stochastic differential equation with fast oscillating coefficients and with respect to a Brownian motion and a Poisson random measure. The large deviation principle of solution is established, and the effect of the highly nonlinear and locally periodic coefficients is stated. Moreover, we derive an explicit expression for the action functional when the viscosity parameter $\varepsilon$ is of order 1 while the homogenization parameter $\delta_{\varepsilon}$ tends to zero.
\end{abstract}

Keywords: Large deviation principle, homogenization, Lévy process, Legendre-Fenchel transform.

2020 MSC: 35B27, 35K57, 60F10, 60H15.

(C)2021 All rights reserved.

\section{Introduction}

The purpose of this paper is to obtain large deviation properties for stochastic differential equations (SDE in short) of Wiener-Poisson type with rapidly fluctuating coefficients and highly oscillating drift. Our method uses the Legendre-Fenchel transform of the limit of normalized logarithm of an exponential moment, as in a periodic case by Freidlin and Sowers [7], and builds on an earlier work by Dupuis and Spiliopoulos [6] which gives us the locally periodic counterpart for SDE of Wiener type. By now, it is well known that stochastic dynamical systems with Poisson jumps are more suitable for capturing sudden bursty fluctuations than classical diffusion models. Motivated by the idea of [6], we consider the locally periodic SDE in the whole space $\mathbb{R}^{\mathrm{d}}$ driven by Lévy noises given by:

$$
\begin{aligned}
X_{t}^{\varepsilon, \delta_{\varepsilon}}-x= & \sqrt{\varepsilon} \int_{0}^{t} \sigma\left(X_{s}^{\varepsilon, \delta_{\varepsilon}}, \frac{X_{s}^{\varepsilon, \delta_{\varepsilon}}}{\delta_{\varepsilon}}\right) d W_{s}+\frac{\varepsilon}{\delta_{\varepsilon}} \int_{0}^{t} b\left(X_{s}^{\varepsilon, \delta_{\varepsilon}}, \frac{X_{s}^{\varepsilon, \delta_{\varepsilon}}}{\delta_{\varepsilon}}\right) d s+\int_{0}^{t} c\left(X_{s}^{\varepsilon, \delta_{\varepsilon}}, \frac{X_{s}^{\varepsilon, \delta_{\varepsilon}}}{\delta_{\varepsilon}}\right) d s \\
& +\int_{0}^{t} \int_{\mathbb{R}_{*}^{d}} k\left(X_{s^{-}}^{\varepsilon, \delta_{\varepsilon}}, \frac{X_{s^{-}}^{\varepsilon, \delta_{\varepsilon}}}{\delta_{\varepsilon}}, u\right)\left(\varepsilon N^{\varepsilon^{-1}}(d s d z)-v(d u) d s\right), x \in \mathbb{R}^{d}, t \geqslant 0,
\end{aligned}
$$

\footnotetext{
${ }^{*}$ Corresponding author

Email addresses: alioune.coulibaly@uam.edu.sn (Alioune Coulibaly), mouhamad.allaya@univ-thies.sn (Mouhamad Mounirou Allaya)
}

doi: $10.22436 /$ jnsa.014.06.06

Received: 2020-06-09 Revised: 2021-03-31 Accepted: 2021-04-07 
according to a given complete Wiener-Poisson space $\left(\Omega, \mathcal{F}, \mathbb{P}, \mathbb{F}, W, N^{\varepsilon^{-1}}\right)$ in $\mathbb{R}^{\mathrm{d}} \times \mathbb{R}_{*}^{\mathrm{d}}, \mathbb{R}_{*}^{\mathrm{d}} \equiv \mathbb{R}^{\mathrm{d}} \backslash\{0\}$, with the Lèvy measure $v$, i.e., $(\Omega, \mathcal{F}, \mathbb{P}, \mathbb{F})$ is a filtered probability space with $\mathbb{F}:=\left\{\mathcal{F}_{t}: t \geqslant 0\right\}$ being the $\mathbb{P}$-completion of the filtration $\mathcal{F},\left\{W_{t}: t \geqslant 0\right\}$ is a d-dimensional standard Brownian motion with respect to $\mathcal{F}_{t}$ and $\mathrm{N}^{\varepsilon^{-1}}$ is a Poisson random counting measure on $\mathbb{R}_{*}^{\mathrm{d}}$ with intensity measure $\varepsilon^{-1} v$, independent of $W$, w.r.t. $\mathcal{F}_{\mathrm{t}}$. In (1.1), $X_{\mathbf{s}^{-}}^{\varepsilon, \delta_{\varepsilon}}$ denotes the left limit, i.e., $\lim _{\substack{r \rightarrow s \\ r<s}} X_{r}^{\varepsilon, \delta_{\varepsilon}}$. Our assumptions on the coefficients $b, c, k$ and $\sigma$ will be specified below.

SDE driven by Lévy processes are widely applied in many fields such as finance, insurance, biology and attracted more and more attention lately (see, [14, 17-19]). Applebaum [1] introduced systematically the definition and relative properties of Lévy processes, and studied the fundamental theory of stochastic integrals such as the Wiener-Lévy-type stochastic integrals and Itô formula, and constructed the framework for the solution and Lévy flow of stochastic ordinary differential equations driven by Lévy processes. Altogether, there are still few results on LDP for stochastic equations with jumps (see for instance $[15,16,21])$. Recently, Manga et al. [10,11] investigated the LDP for periodic stochastic equation driven by small multiple Lévy noises, as they pointed out in $[10,11]$, that the difficulties caused by Lévy noises are to deal with the analysis of the logarithmic moment generating function.

Baldi [2] was the first to consider a family of periodic diffusion processes with homogenization and a small multiplicative parameter of the diffusion coefficient. He established a large deviation principle and as an application, he derived an iterated logarithm law for periodic diffusions. Afterwards, Freidlin and Sowers [7] studied the combined effects of homogenization and large deviations in a stochastic differential equation. The authors showed some large deviations type estimates, and then applied these results to study wavefronts both in a single reaction-diffusion equation and in a system of reaction-diffusion equations. Apart from [7], LDP for stochastic equation depending on the coefficients of the system on the slow and fast motion, has also been studied in $[9,20]$ under various assumptions. It is necessary to point out that Dupuis and Spiliopoulos [6] just considered LDP for Wiener equation type with locally periodic coefficients. In [6] large deviations problems arising in locally periodic stochastic equation with small noise and fast oscillating coefficients are discussed, and the authors used methods from weak convergence and stochastic control. Such results are not available in the literature. The scope of our approach based on the LDP with with locally periodic coefficients, differs substantially from that of [6]. Our version of SDE contains a substantial novelty, which makes it possible to handle the large deviation arising in stochastic homogenization for nonlinear equations with Poisson jumps and Brownian motions. In the present paper, we have derived the same explicit expression for the action functional in [6, Regime 1] via Legendre-Fenchel transform.

The paper is organized as follows. In Section 2 we set up some notations, precise our assumptions and give away the toolbox for the LDP characterization. In Section 3 we study large deviations for $X_{\mathrm{T}}^{\varepsilon, \delta_{\varepsilon}}$ with fixed $T>0$, as $\varepsilon$ and $\delta_{\varepsilon}$ both tending to zero.

\section{Preliminaries}

\subsection{Notations and assumptions}

Let's denote by $\mathbb{E}$ the expectation with respect to $\mathbb{P}$. Define $\langle.,$.$\rangle as the standard Euclidean inner$ product on $\mathbb{R}^{\mathrm{d}}$, and $\|\cdot\|$ as the associated norm. For each $z \in \mathbb{R}^{\mathrm{d}}$, let $\mathrm{a}^{-1}(z)$ be the matrix inverse of $a(z)$, when $\mathrm{a}^{-1}$ is well-defined on all of $\mathbb{R}^{\mathrm{d}}$. For each $z \in \mathbb{R}^{\mathrm{d}}$ define the norm $\|\theta\|_{\mathrm{a}^{-1}(z)}=\sqrt{\left\langle\theta, \mathrm{a}^{-1}(z) \theta\right\rangle}$ for all $\theta \in \mathbb{R}^{\mathrm{d}}$. Let $\mathcal{D}\left([0, T], \mathbb{R}^{\mathrm{d}}\right)$ be the space of functions that map $[0, T]$ into $\mathbb{R}^{\mathrm{d}}$, which are right continuous and having left hand limits.

Condition 2.1. We assume that $\lim _{\varepsilon \rightarrow 0} \frac{\delta_{\varepsilon}}{\varepsilon}=0$ (main hypothesis).

In preparation for stating the main results, we recall the concept of large deviation principle. 
Definition 2.2. Let $X^{\varepsilon, \delta_{\varepsilon}}$ be a $\mathbb{R}^{\mathrm{d}}$-valued random variable and let $\mathbb{P}_{\varepsilon, \delta_{\varepsilon}}$ denote its distribution on the Borel subsets of $\mathbb{R}^{\mathrm{d}}$, that is, $\mathbb{P}_{\varepsilon, \delta_{\varepsilon}}(A)=\mathbb{P}\left(X^{\varepsilon, \delta_{\varepsilon}} \in A\right)$. The family $\left\{X^{\varepsilon, \delta_{\varepsilon}}\right\}_{\varepsilon, \delta_{\varepsilon} \geqslant 0}$ satisfies an LDP if there exists a lower semi-continuous function $\mathrm{I}: \mathbb{R}^{\mathrm{d}} \longrightarrow[0,+\infty]$ such that

- for each open set $O \subseteq \mathbb{R}^{\mathrm{d}}, \liminf _{\varepsilon \rightarrow 0} \varepsilon \log \mathbb{P}\left(X^{\varepsilon, \delta_{\varepsilon}} \in \mathrm{O}\right) \geqslant-\inf _{x \in O} \mathrm{I}(x)$;

- for each closed set $F \subseteq \mathbb{R}^{d}, \limsup _{\varepsilon \rightarrow 0} \varepsilon \log \mathbb{P}\left(X^{\varepsilon, \delta_{\varepsilon}} \in F\right) \leqslant-\inf _{x \in F} I(x)$.

I is called the rate function for the LDP. A rate function is good if for each $a \in \mathbb{R}_{+},\{x: I(x) \leqslant a\}$ is compact.

Regarding SDE (1.1), we impose the following conditions.

\section{Condition 2.3.}

(H.1) The functions $b, c: \mathbb{R}^{\mathrm{d}} \times \mathbb{R}^{\mathrm{d}} \longmapsto \mathbb{R}^{\mathrm{d}}, \sigma: \mathbb{R}^{\mathrm{d}} \times \mathbb{R}^{\mathrm{d}} \longmapsto \mathbb{R}^{\mathrm{d} \times \mathrm{d}}$ are Lipschitz continuous and bounded in $(x, y)$ and periodic with period 1 with respect to $y$ in each direction. We additionally assume that they are $C^{1}\left(\mathbb{R}^{d}\right)$ in $y$ and $C^{2}\left(\mathbb{R}^{d}\right)$ in $x$ with all partial derivatives continuous and globally bounded in $x$ and $y$. The function $k: \mathbb{R}^{\mathrm{d}} \times \mathbb{R}^{\mathrm{d}} \times \mathbb{R}^{\mathrm{d}} \longmapsto \mathbb{R}^{\mathrm{d}}$ is Lipschitz continuous and bounded in $(x, y)$ and periodic with period 1 with respect to $y$ in each direction, and integrable with respect to dtdu.

(H.2) The diffusion matrix $a:=\sigma \sigma^{*}$ is uniformly non-degenerate.

We define some family of operators indexed by $x$ (resp. $y$ ) and acting on the second argument (resp. the first argument)

$$
\begin{aligned}
\mathcal{L}_{\varepsilon, \delta_{\varepsilon}}^{x} & =\frac{\varepsilon}{2} \sum_{i, j=1}^{d} a_{i j}\left(x, \frac{y}{\delta_{\varepsilon}}\right) \frac{\partial^{2}}{\partial y_{i} \partial y_{j}}+\frac{\varepsilon}{\delta_{\varepsilon}} \sum_{i=1}^{d} b_{i}\left(x, \frac{y}{\delta_{\varepsilon}}\right) \frac{\partial}{\partial y_{i}}+\sum_{i=1}^{d} c_{i}\left(x, \frac{y}{\delta_{\varepsilon}}\right) \frac{\partial}{\partial y_{i}} \\
\mathcal{L}_{\varepsilon, \delta_{\varepsilon}}^{y} & =\frac{\varepsilon}{2} \sum_{i, j=1}^{d} a_{i j}\left(x, \frac{y}{\delta_{\varepsilon}}\right) \frac{\partial^{2}}{\partial x_{i} \partial x_{j}}+\frac{\varepsilon}{\delta_{\varepsilon}} \sum_{i=1}^{d} b_{i}\left(x, \frac{y}{\delta_{\varepsilon}}\right) \frac{\partial}{\partial x_{i}}+\sum_{i=1}^{d} c_{i}\left(x, \frac{y}{\delta_{\varepsilon}}\right) \frac{\partial}{\partial x_{i}}, \\
\mathcal{L}_{x} & =\frac{1}{2} \sum_{i, j=1}^{d} a_{i j}(x, y) \frac{\partial^{2}}{\partial y_{i} \partial y_{j}}+\sum_{i=1}^{d} b_{i}(x, y) \frac{\partial}{\partial y_{i}} .
\end{aligned}
$$

With our requirement, there exists a Lèvy process on $\mathbb{R}^{\mathrm{d}}$ with diffusion-generator $\mathcal{L}_{\mathrm{X}}$ and by $\mathrm{Y}$ periodicity assumption on the coefficients such a process induces a Wiener-Poisson process $\bar{Y}^{x}$ on the $\mathrm{d}$-dimensional torus $\mathbb{T}^{\mathrm{d}}$, moreover this diffusion process part is ergodic. We denote by $m(x,$.$) its unique$ invariant measure. In order for the process with diffusion-generator $\mathcal{L}_{\varepsilon, \delta_{\varepsilon}}^{x}$ to have a limit in law as $\varepsilon \rightarrow 0$, we need that the following condition be enforced.

\section{Condition 2.4.}

(H.3) For all $x \in \mathbb{R}^{\mathrm{d}}, \int_{\mathbb{T}^{\mathrm{d}}} \mathrm{b}(x, y) \mathrm{m}(x, \mathrm{~d} y)=0$ (centering condition).

We notice that, under the Condition 2.4, there exists a unique periodic solution $\hat{b}^{i}$ of $\mathcal{L}_{x} \hat{b}^{i}-b^{i}=0$, for any $i=1, \ldots, d$ with zero integral against the measure $m(x,$.$) . Such a solution is given by the formula$ $\hat{b}^{i}(x, y)=\int_{0}^{\infty} \mathbb{E}_{y}\left\{b\left(x, \bar{Y}_{t}^{x}\right)\right\} d t$ where under $\mathbb{P}_{y}, \bar{Y}^{x}$ starts from $y$. Moreover $\hat{b}$ is twice continuously differentiable in $x$, uniformly with respect to $y$, and for any $p \geqslant 2$ there exists a constant $K>0$ such that for all $x \in \mathbb{R}^{\mathrm{d}}$ (see, Benchérif-Madani and Pardoux [4] or Pardoux and Veretennikov [12, 13])

$$
\left\{\|\hat{b}\|_{W^{2, p}\left(\mathbb{T}^{d}\right)}+\left\|\frac{\partial \hat{b}}{\partial x}\right\|_{W^{2, p}\left(\mathbb{T}^{d}\right)}+\left\|\frac{\partial \hat{b}}{\partial y}\right\|_{W^{2, p}\left(\mathbb{T}^{d}\right)}+\left\|\frac{\partial^{2} \hat{b}}{\partial x^{2}}\right\|_{W^{2, p}\left(\mathbb{T}^{d}\right)}+\left\|\frac{\partial^{2} \hat{b}}{\partial x \partial y}\right\|_{W^{2, p}\left(\mathbb{T}^{d}\right)}\right\}(x, .) \leqslant K .
$$


Let's set $\bar{X}_{t}^{\varepsilon, \delta_{\varepsilon}}:=X_{\left(\delta_{\varepsilon} / \sqrt{\varepsilon}\right)^{2} \delta^{2}}$. In order to move the SDE (1.1) into the torus $\mathbb{T}^{\mathrm{d}}$, we define the pull-back $\bar{Y}_{\mathrm{t}}^{\varepsilon, \delta_{\varepsilon}}:=\frac{1}{\delta_{\varepsilon}} \bar{X}_{\mathrm{t}}^{\varepsilon, \delta_{\varepsilon}}$, which satisfies the SDE :

$$
\begin{aligned}
\bar{Y}_{t}^{\varepsilon, \delta}-\frac{x}{\delta_{\varepsilon}}= & \int_{0}^{t} \sigma\left(\bar{X}_{s}^{\varepsilon, \delta_{\varepsilon}}, \bar{Y}_{s}^{\varepsilon, \delta_{\varepsilon}}\right) d \bar{W}_{s}^{\varepsilon}+\int_{0}^{t} b\left(\bar{X}_{s}^{\varepsilon, \delta_{\varepsilon}}, \bar{Y}_{s}^{\varepsilon, \delta_{\varepsilon}}\right) d s \\
& +\frac{\delta_{\varepsilon}}{\varepsilon} \int_{0}^{t} c\left(\bar{X}_{s}^{\varepsilon, \delta_{\varepsilon}}, \bar{Y}_{s}^{\varepsilon, \delta_{\varepsilon}}\right) d s+\int_{0}^{t} \int_{\mathbb{R}_{*}^{d}} k\left(\bar{X}_{s^{-}}^{\varepsilon, \delta_{\varepsilon}}, \bar{Y}_{s^{-}}^{\varepsilon, \delta_{\varepsilon}}, u\right) d \bar{M}_{s, u}^{\varepsilon},
\end{aligned}
$$

where

$$
\begin{aligned}
\bar{W}_{\mathrm{t}}^{\varepsilon} & :=\frac{\sqrt{\varepsilon}}{\delta_{\varepsilon}} W_{\left(\delta_{\varepsilon} / \sqrt{\varepsilon}\right)^{2} \mathrm{t}} \text { is Brownian motion, } \\
\bar{M}_{\mathrm{t}, \mathrm{u}}^{\varepsilon} & :=\int_{0}^{\mathrm{t}}\left(\mathrm{N}^{\left(\delta_{\varepsilon} / \varepsilon\right)}(\mathrm{d} s \mathrm{~d} u)-\frac{\delta_{\varepsilon}}{\varepsilon} v(\mathrm{du}) \mathrm{ds}\right) \text { is a Poissonian martingale. }
\end{aligned}
$$

The infinitesimal generator $\overline{\mathcal{L}}_{\varepsilon, \delta_{\varepsilon}}^{x}$ of the diffusion part associated to the process $\bar{Y}^{\varepsilon, \delta_{\varepsilon}}$ is

$$
\overline{\mathcal{L}}_{\varepsilon, \delta_{\varepsilon}}^{x}:=\frac{1}{2} \sum_{i, j=1}^{d} a_{i j}(x, y) \frac{\partial^{2}}{\partial y_{i} \partial y_{j}}+\sum_{i=1}^{d} b_{i}(x, y) \frac{\partial}{\partial y_{i}}+\frac{\delta_{\varepsilon}}{\varepsilon} \sum_{i=1}^{d} c_{i}(x, y) \frac{\partial}{\partial y_{i}}, \quad x \in \mathbb{R}^{d}, y \in \mathbb{T}^{d} .
$$

This generator tends to $\mathcal{L}_{x}$ defined as (2.1), when $\varepsilon \rightarrow 0$.

\subsection{Used lemmas}

The proof of the main Theorem 3.1 (below) relies on explicit calculation of the logarithm moments of $X^{\varepsilon, \delta_{\varepsilon}}$ and the following Girsanov's formula and Itô's formula. Before proceeding, let us introduce some spaces.

For $E$ locally compact, let $\mathcal{H}^{2}(T, \lambda)$ be the linear space of all equivalence classes of mappings $F$ : $[0, \mathrm{~T}] \times \mathrm{E} \times \Omega \longrightarrow \mathbb{R}$ which coincide almost everywhere with respect to $\mathrm{dt} \otimes \mathrm{d} \lambda \otimes \mathrm{dP}$ and which satisfy the following conditions :

- $\mathrm{F}$ is predictable;

- $\int_{0}^{T} \int_{E \backslash\{0\}} \mathbb{E}\left(|F(t, z)|^{2}\right) d t \lambda(d z)<+\infty$.

We endow $\mathcal{H}^{2}(T, \lambda)$ with the inner product $\langle F, G\rangle_{T, \lambda}:=\int_{0}^{T} \int_{E \backslash\{0\}} \mathbb{E}(F(t, z) G(t, z)) d t \lambda(d z)$. Then, it is well known that $\left(\mathcal{H}^{2}(T, \lambda) ;\langle.,\rangle_{T, \lambda}\right)$ is a real separable Hilbert space.

Let $N_{p}$ be a Poisson random measure on $\mathbb{R}_{+} \times(E \backslash\{0\})$ with intensity measure $\lambda$, according to a given

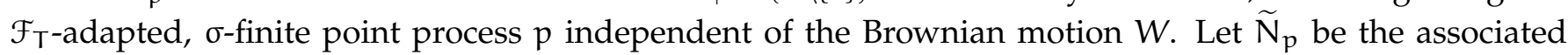
compensated Poisson random measure. Now we have following (see, Applebaum [1] Chapter 5, Section 2).

Lemma 2.5 (Girsanov's formula). Let $\mathrm{X}$ be a Lèry process such that $\mathrm{e}^{\mathrm{X}}$ is a martingale, i.e.,

$$
X_{t}=\int_{0}^{t} b(s) d s+\int_{0}^{t} \sigma(s) d W_{s}+\int_{0}^{t} \int_{E} H(s, z) \widetilde{N}_{p}(d s d z)+\int_{0}^{t} \int_{E} K(s, z) N_{p}(d s d z)
$$

with

$$
b(t)=-\frac{1}{2} \sigma^{2}(t)-\int_{E}\left(e^{H(t, z)}-1-H(t, z)\right) \lambda(d z)-\int_{E}\left(e^{K(t, z)}-1\right) \lambda(d z), \mathbb{P}-\text { a.s. }
$$


We suppose that there exists $\mathrm{C}>0$ such that

$$
|\mathrm{K}(\mathrm{t}, z)| \leqslant \mathrm{C}, \quad \forall \mathrm{t} \geqslant 0, \forall z \in \mathrm{E} .
$$

For $\mathrm{L} \in \mathcal{H}^{2}(\mathrm{~T}, \lambda)$ we define

$$
M_{\mathrm{t}}:=\int_{0}^{\mathrm{t}} \int_{z \neq 0} \mathrm{~L}(s, z) \widetilde{\mathrm{N}}(\mathrm{d} s \mathrm{~d} z)
$$

Set

$$
\mathrm{U}(\mathrm{t}, z)=\left(\mathrm{e}^{\mathrm{H}(\mathrm{t}, z)}-1\right) \mathbf{1}_{\{\|z\|<1\}}+\left(\mathrm{e}^{\mathrm{K}(\mathrm{t}, z)}-1\right) \mathbf{1}_{\{\|z\| \geqslant 1\}}
$$

and we suppose that

$$
\int_{0}^{T} \int_{\{\|z\| \leqslant 1\}}\left(e^{H(s, z)}-1\right)^{2} \lambda(\mathrm{d} z) \mathrm{d} s<+\infty
$$

Finally, we define

$$
\mathrm{B}_{\mathrm{t}}=\mathrm{W}_{\mathrm{t}}-\int_{0}^{\mathrm{t}} \sigma(\mathrm{s}) \mathrm{ds} \text { and } \mathrm{N}_{\mathrm{t}}=\mathrm{M}_{\mathrm{t}}-\int_{0}^{\mathrm{t}} \int_{z \neq 0} \mathrm{~L}(\mathrm{~s}, z) \mathrm{U}(\mathrm{s}, z) \lambda(\mathrm{d} z) \mathrm{ds}, 0 \leqslant \mathrm{t} \leqslant \mathrm{T} .
$$

Let $\mathbf{Q}$ be the probability measure on $\left(\Omega, \mathcal{F}_{\mathrm{T}}\right)$ defined as:

$$
\frac{\mathrm{dQ}}{\mathrm{dP}}:=e^{\mathrm{X}_{\mathrm{T}}} .
$$

Then under $\mathrm{Q}, \mathrm{B}_{\mathrm{t}}$ is a Brownian motion and $\mathrm{N}_{\mathrm{t}}$ is a $\mathrm{Q}$-martingale.

Next, define a d-dimensional semi-martingale $Y_{t}:=\left(Y_{1}, \ldots, Y_{d}\right)$ by

$$
Y_{t}^{i}=Y_{0}^{i}+M_{t}^{i}+A_{t}^{i}+\int_{0}^{t} \int_{E} f_{i}(s, z, .) \widetilde{N}_{p}(d s d z)+\int_{0}^{t} \int_{E} g_{i}(s, z, .) N_{p}(d s d z), \quad i:=1, \ldots, d,
$$

where

- $\mathrm{M}_{\mathrm{t}}$ is locally continuous square integrable $\left(\mathcal{F}_{\mathrm{t}}\right)$-martingale and $\mathrm{M}_{0}:=0$;

- $A_{t}$ is a continuous $\left(\mathcal{F}_{t}\right)$-adapted process whose almost all sample functions are of bounded variation on each finite interval and $A_{0}:=0$;

- $g$ is $\left(\mathcal{F}_{\mathrm{t}}\right)$-predictable and for $\mathrm{t}>0, \int_{0}^{\mathrm{t}} \int_{\mathrm{E}}\|g(s, z,).\| \lambda(\mathrm{d} z) \mathrm{d} s<+\infty$ a.s;

- $f$ is $\left(\mathcal{F}_{t}\right)$-predictable and for $t>0, \int_{0}^{t} \int_{E}\|f(s, z, .)\|^{2} \lambda(d z) d s<+\infty$ a.s.

We have following (see, for example Ikeda and Watanabe [8], Theorem 5.1).

Lemma 2.6 (Itô's formula). Let $\mathrm{F}$ be a function of class $\mathrm{C}^{2}$ on $\mathbb{R}^{\mathrm{d}}$ and $\mathrm{Y}_{\mathrm{t}}$ a d-dimensional semi-martingale given in (2.2). Then the stochastic process $F\left(Y_{t}\right)$ is also a $\left(\mathcal{F}_{t}\right)$-semi-martingale and the following formula holds:

$$
\begin{aligned}
F\left(Y_{t}\right)-F\left(Y_{0}\right)= & \sum_{i=1}^{d} \int_{0}^{t} \frac{\partial F}{\partial x_{i}}\left(Y_{s}\right) d M_{s}^{i}+\sum_{i=1}^{d} \int_{0}^{t} \frac{\partial F}{\partial x_{i}}\left(Y_{s}\right) A_{s}^{i} d s+\frac{1}{2} \sum_{i, j=1}^{d} \int_{0}^{t} \frac{\partial^{2} F}{\partial x_{i} \partial x_{j}}\left(Y_{s}\right) d\left\langle M^{i}, M^{j}\right\rangle_{s} \\
& +\int_{0}^{t} \int_{E}\left[F\left(Y_{s^{-}}+g(s, z, .)\right)-F\left(Y_{s^{-}}\right)\right] N_{p}(d z d s) \\
& +\int_{0}^{t} \int_{E}\left[F\left(Y_{s^{-}}+f(s, z, .)\right)-F\left(Y_{s^{-}}\right)\right] \widetilde{N}_{p}(d z d s) \\
& +\int_{0}^{t} \int_{E}\left[F\left(Y_{s^{-}}+f(s, z, .)\right)-F\left(Y_{s^{-}}\right)-\sum_{i=1}^{d} f_{i}(s, z, .) \frac{\partial F}{\partial x_{i}}\left(Y_{s}\right)\right] \lambda(d z) d s .
\end{aligned}
$$


The next Lemma is the main technique for showing that $X^{\varepsilon, \delta_{\varepsilon}}$ has LDP. For all $\mathrm{T}>0$ and $x \in \mathbb{R}^{\mathrm{d}}$, set

$$
g_{T, x}^{\varepsilon}(\theta):=\varepsilon \log \mathbb{E}\left\{\exp \left(\frac{1}{\varepsilon}\left\langle\theta, X_{T}^{\chi, \varepsilon}, \delta_{\varepsilon}\right\rangle\right)\right\}, \quad \varepsilon>0, \theta \in \mathbb{R}^{\mathrm{d}} .
$$

Let us set

$$
\mathrm{g}_{\mathrm{T}, \mathrm{x}}(\theta):=\lim _{\mathcal{\varepsilon} \rightarrow 0} \mathrm{~g}_{\mathrm{T}, \mathrm{\varepsilon}}^{\varepsilon}(\theta), \quad \theta \in \mathbb{R}^{\mathrm{d}},
$$

when this limit exists. We then have following (see, Dembo and Zeitouni [5], Chap. 2.3).

Lemma 2.7 (Gärtner-Ellis theorem). Fix $\mathrm{T}>0$ and $\theta \in \mathbb{R}^{\mathrm{d}}$. Assume that

i.) for each $\theta \in \mathbb{R}^{\mathrm{d}}, \mathrm{g}_{\mathrm{T}, \mathrm{x}}$ is well-defined in $[-\infty,+\infty]$;

ii.) the origin is in the interior of the set $\left\{\theta \in \mathbb{R}^{\mathrm{d}}: \mathrm{g}_{\mathrm{T}, \mathrm{x}}(\theta)<+\infty\right\}$;

iii.) the set $A:=\left\{\theta \in \mathbb{R}^{\mathrm{d}}:\left|\mathrm{g}_{\mathrm{T}, \mathrm{x}}(\theta)\right|<+\infty\right\}$ has no empty interior $\mathrm{A}^{\circ}, \nabla \mathrm{g}_{\mathrm{T}, \mathrm{x}}(\theta)$ is well-defined for all $\theta \in \mathrm{A}^{\circ}$ and

$$
\limsup _{\substack{\theta \rightarrow \partial A \\ \theta \in A^{\circ}}}\left\|\nabla g_{T, x}(\theta)\right\|=+\infty
$$

Then the random variable $\left\{\mathrm{X}_{\mathrm{T}}^{\varepsilon, \delta_{\varepsilon}}: \varepsilon>0\right\}$ has an LDP with rate function $\mathrm{I}_{\mathrm{T}, \mathrm{x}}$ defined as

$$
\mathrm{I}_{\mathrm{T}, x}(z):=\sup _{\theta \in \mathbb{R}^{\mathrm{d}}}\left\{\langle\theta, z\rangle-\mathrm{g}_{\mathrm{T}, \mathrm{x}}(\theta)\right\} .
$$

\section{Large deviation and discussion}

In this section, we will apply the generalized inequality of derivation from convex analysis (Lemma 2.7, Gärtner-Ellis theorem) of large deviation to show the solution of stochastic equation with jumps (1.1) satisfies the LDP. For this, we proceed in a similar way to that used in Freidlin and Sowers [7]. The main difficulty which arises is that in SDE (1.1) the coefficients are not supposed to be periodic, and furthermore the stochastic equation is not governed by a continuous process. This difficulty is completely technical in nature, and it is resolved by choosing a suitable change of probabilities (Lemma 2.5, Girsanov's formula) or of variables (Lemma 2.6, Itô's formula).

\subsection{Main result}

Before proceeding, let us define

$$
\begin{aligned}
\hat{b} & :=\left[\begin{array}{c}
\hat{b}^{1} \\
\vdots \\
\hat{b}^{d}
\end{array}\right] \quad \text { and } \nabla_{u} \hat{b}:=\left[\begin{array}{llr}
\frac{\partial \hat{b}^{1}}{\partial u_{1}} & \cdots & \frac{\partial \hat{b}^{1}}{\partial u_{d}} \\
\vdots & \cdots & \vdots \\
\frac{\partial \hat{b}^{d}}{\partial u_{1}} & \cdots & \frac{\partial \hat{b}^{d}}{\partial u_{d}}
\end{array}\right] \text { for each } u=x, y ; \\
\bar{a}(x) & :=\int_{\mathbb{T}^{d}}\left[\left(I+\nabla_{y} \hat{b}\right) a\left(I+\nabla_{y} \hat{b}\right)^{*}\right](x, y) m(x, d y) \\
\bar{c}(x) & :=\int_{\mathbb{T}^{d}}\left[\left(I+\nabla_{y} \hat{b}\right) c+\operatorname{Tr}\left\{a\left(\nabla_{x} \nabla_{y} \hat{b}\right)\right\}\right](x, y) m(x, d y) \\
\bar{k}(x) & :=\int_{\mathbb{R}_{*}^{d}}\left[\left(I+\nabla_{y} \hat{b}\right)(x, y) k(x, y, u)\right] m(x, d y) v(d u) .
\end{aligned}
$$

Next, we state our main result.

Theorem 3.1 (Main result). Fix $\mathrm{T}>0$ and assume that the conditions 2.1, 2.3, and 2.4 remain valid. Then for 


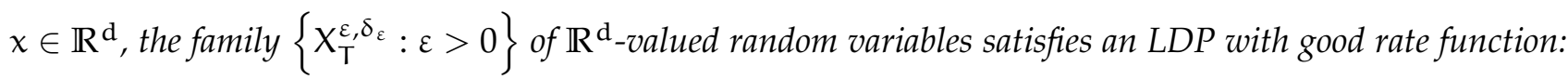

$$
\mathrm{I}_{\mathrm{T}, x}(z):=\mathrm{T}_{1}\left(\frac{z-x}{\mathrm{~T}}\right)+\mathrm{T}_{2}\left(\frac{z-x}{\mathrm{~T}}\right), \quad z \in \mathbb{R}^{\mathrm{d}},
$$

where

$$
\begin{aligned}
& J_{1}(z):=\inf _{\substack{\varphi \in \mathcal{D}\left([0, \mathrm{~T}], \mathbb{R}^{\mathrm{d}}\right) \\
\varphi_{0}=x}} \frac{1}{2} \int_{0}^{\mathrm{T}}\left\|z-\overline{\mathrm{c}}\left(\varphi_{\mathrm{s}}\right)+\overline{\mathrm{k}}\left(\varphi_{\mathrm{s}}\right)\right\|_{(\overline{\mathrm{a}})^{-1}\left(\varphi_{\mathrm{s}}\right)}^{2} \mathrm{ds}, \\
& \partial_{2}(z):=\inf _{\substack{\varphi \in \mathcal{D}\left([0, \mathrm{~T}], \mathbb{R}^{\mathrm{d}}\right) \\
\varphi_{0}=x}} \int_{0}^{T} \int_{\mathbb{R}_{*}^{\mathrm{d}}} \int_{\mathbb{T}^{\mathrm{d}}} \rho\left(\frac{\|z\|}{\left\|\mathrm{k}\left(\varphi_{\mathrm{s}^{-}}, \mathrm{y}, \mathrm{u}\right)\right\|}\right) \mathrm{m}(x, \mathrm{~d} y) v(\mathrm{du}) \mathrm{ds},
\end{aligned}
$$

and where $\rho(r):=r \log r-r+1, \quad r \in(0,+\infty)$.

\subsection{Proof of the main result}

Proof of Theorem 3.1. Let's us set $\hat{Y}_{t}^{\varepsilon, \delta_{\varepsilon}}:=\frac{1}{\delta_{\varepsilon}} X_{t}^{\varepsilon, \delta_{\varepsilon}}$, we have by Itô's formula (Lemma 2.6) that,

$$
\begin{aligned}
& \tilde{X}_{t}^{\varepsilon, \delta_{\varepsilon}}=X_{t}^{\varepsilon, \delta_{\varepsilon}}+\delta_{\varepsilon}\left[\hat{b}\left(X_{t}^{\varepsilon, \delta_{\varepsilon}}, \hat{Y}_{t}^{\varepsilon, \delta_{\varepsilon}}\right)-\hat{b}\left(x, \frac{x}{\delta_{\varepsilon}}\right)\right] \\
& =x+\sqrt{\varepsilon} \int_{0}^{t}\left(I+\nabla_{y} \hat{b}+\delta_{\varepsilon} \nabla_{x} \hat{b}\right)\left(X_{s}^{\varepsilon, \delta_{\varepsilon}}, \hat{Y}_{s}^{\varepsilon, \delta_{\varepsilon}}\right) \sigma\left(X_{s}^{\varepsilon, \delta_{\varepsilon}}, \hat{Y}_{s}^{\varepsilon, \delta_{\varepsilon}}\right) d W_{s} \\
& +\int_{0}^{t}\left(I+\nabla_{y} \hat{b}\right)\left(X_{s}^{\varepsilon, \delta_{\varepsilon}}, \hat{Y}_{s}^{\varepsilon, \delta_{\varepsilon}}\right) c\left(X_{s}^{\varepsilon, \delta_{\varepsilon}}, \hat{Y}_{s}^{\varepsilon, \delta_{\varepsilon}}\right) d s+\delta_{\varepsilon} \int_{0}^{t} \mathcal{L}_{\varepsilon, \delta_{\varepsilon}}^{\hat{Y}_{\varepsilon}^{,} \delta_{\varepsilon}} \hat{b}\left(X_{s}^{\varepsilon, \delta_{\varepsilon}}, \hat{Y}_{s}^{\varepsilon, \delta_{\varepsilon}}\right) d s \\
& +\int_{0}^{t} \operatorname{Tr}\left\{a\left(X_{s}^{\varepsilon, \delta_{\varepsilon}}, \hat{Y}_{s}^{\varepsilon, \delta_{\varepsilon}}\right)\left(\nabla_{x} \nabla_{y} \hat{b}\right)\left(X_{s}^{\varepsilon, \delta_{\varepsilon}}, \hat{Y}_{s}^{\varepsilon, \delta_{\varepsilon}}\right)\right\} d s-\int_{0}^{t} \int_{\mathbb{R}_{*}^{d}} k\left(X_{s^{-}}^{\varepsilon, \delta_{\varepsilon}}, \hat{Y}_{s^{-}}^{\varepsilon, \delta_{\varepsilon}}, u\right) v(d u) d s \\
& -\int_{0}^{t} \int_{\mathbb{R}_{*}^{d}}\left(\delta_{\varepsilon} \nabla_{x} \hat{b}+\nabla_{y} \hat{b}\right)\left(X_{s}^{\varepsilon, \delta_{\varepsilon}}, \hat{Y}_{s}^{\varepsilon, \delta_{\varepsilon}}\right) k\left(X_{s^{-}}^{\varepsilon, \delta_{\varepsilon}}, \hat{Y}_{s^{-}}^{\varepsilon, \delta_{\varepsilon}}, u\right) v(d u) d s \\
& +\delta_{\varepsilon} \int_{0}^{t} \int_{\mathbb{R}_{*}^{d}}\left[\hat{b}\left(X_{s^{-}}^{\varepsilon, \delta_{\varepsilon}}+\varepsilon k\left(X_{s^{-}}^{\varepsilon, \delta_{\varepsilon}}, \hat{Y}_{s^{-}}^{\varepsilon, \delta_{\varepsilon}}, u\right), \hat{Y}_{s^{-}}^{\varepsilon, \delta_{\varepsilon}}, u\right)-\hat{b}\left(X_{s^{-}}^{\varepsilon, \delta_{\varepsilon}}, \hat{Y}_{s^{-}}^{\varepsilon, \delta_{\varepsilon}}\right)\right] N^{\varepsilon^{-1}}(\mathrm{duds}) \\
& +\delta_{\varepsilon} \int_{0}^{t} \int_{\mathbb{R}_{*}^{d}}\left[\hat{b}\left(X_{s^{-}}^{\varepsilon, \delta_{\varepsilon}}, \hat{Y}_{s^{-}}^{\varepsilon, \delta_{\varepsilon}}+\varepsilon k\left(X_{s^{-}}^{\varepsilon, \delta_{\varepsilon}}, \hat{Y}_{s^{-}}^{\varepsilon, \delta_{\varepsilon}}, u\right), u\right)-\hat{b}\left(X_{s^{-}}^{\varepsilon, \delta_{\varepsilon}}, \hat{Y}_{s^{-}}^{\varepsilon, \delta_{\varepsilon}}\right)\right] N^{\varepsilon^{-1}}(\mathrm{duds}) \\
& +\varepsilon \int_{0}^{t} \int_{\mathbb{R}_{*}^{d}} k\left(X_{s^{-}}^{\varepsilon, \delta_{\varepsilon}}, \hat{Y}_{s^{-}}^{\varepsilon, \delta_{\varepsilon}}, u\right) N^{\varepsilon^{-1}}(\mathrm{~d} u d s) .
\end{aligned}
$$

Let us define for $(x, y) \in \mathbb{R}^{\mathrm{d}} \times \mathbb{T}^{\mathrm{d}}$,

$$
H^{\varepsilon, \psi}(x, y):=\psi(x+\varepsilon k(x, y, .), y)+\psi(x, y+\varepsilon k(x, y, .))-2 \psi(x, y) \quad \forall \psi \in C^{2,1}\left(\mathbb{R}^{d}, \mathbb{T}^{d}\right) .
$$

So, we are going to consider the logarithm moment generating function $g_{t, x}^{\varepsilon}$ (2.3) of $\tilde{X}^{\varepsilon, \delta_{\varepsilon}}$ given by (3.2). In order to lighten the formulas, let $\xi$ denote any of coefficients $a, b, c, k$. Even if the coefficients $a, b$ and $c$ do not depend on $z$ we still allow for the notation $\xi(r, r, u)$ meaning, respectively, $\xi\left(\bar{X}_{r}^{\varepsilon, \delta_{\varepsilon}}, \bar{Y}_{r}^{\varepsilon, \delta_{\varepsilon}}\right)$ and $\xi\left(\bar{X}_{r}^{\varepsilon, \delta_{\varepsilon}}, \bar{Y}_{r}^{\varepsilon, \delta_{\varepsilon}}, u\right)$, for each $r=s, t, \frac{\varepsilon}{\delta_{\varepsilon}^{2}}$ t. There should be no confusion with space variables since we use different letters for these arguments. Thus we have by Girsanov's formula (Lemma 2.5) that

$$
g_{\mathrm{t}, x}^{\varepsilon}(\theta)=\langle x, \theta\rangle+\varepsilon \log \widetilde{\mathbb{E}}\left[\exp \left\{\left(\frac{\delta_{\varepsilon}}{\varepsilon}\right)^{2} \int_{0}^{\left(\sqrt{\varepsilon} / \delta_{\varepsilon}\right)^{2} \mathrm{t}} \Phi^{\varepsilon, \theta}(s, s) \mathrm{d} s-\frac{\delta_{\varepsilon}}{\varepsilon}\left[\hat{b}\left(\frac{\varepsilon}{\delta_{\varepsilon}^{2}} \mathrm{t}, \frac{\varepsilon}{\delta_{\varepsilon}^{2}} \mathrm{t}\right)-\hat{b}\left(x, \frac{x}{\delta_{\varepsilon}}\right)\right]\right\}\right],
$$


where

$$
\begin{aligned}
\Phi^{\varepsilon, \theta}(\mathrm{t}, \mathrm{t}):= & \frac{1}{2}\left\langle\theta,\left(\mathrm{I}+\nabla_{y} \hat{\mathrm{b}}+\delta_{\varepsilon} \nabla_{x} \hat{\mathrm{b}}\right) \sigma(\mathrm{t}, \mathrm{t})\right\rangle^{2}+\left\langle\theta,\left(\mathrm{I}+\nabla_{y} \hat{\mathrm{b}}\right) \mathrm{c}(\mathrm{t}, \mathrm{t})\right\rangle+\left\langle\theta, \operatorname{Tr}\left\{\mathrm{a}\left(\nabla_{x} \nabla_{y} \hat{\mathrm{b}}\right)(\mathrm{t}, \mathrm{t})\right\}\right\rangle \\
& -\int_{\mathbb{R}_{*}^{d}}\left\langle\theta,\left(\mathrm{I}+\delta_{\varepsilon} \nabla_{x} \hat{\mathrm{b}}+\nabla_{y} \hat{\mathrm{b}}\right) \mathrm{k}(\mathrm{t}, \mathrm{t}, \mathrm{u})\right\rangle v(\mathrm{du})+\delta_{\varepsilon}\left\langle\theta, \mathcal{L}_{\varepsilon, \delta_{\varepsilon}}^{\bar{\varepsilon}_{\varepsilon}^{\varepsilon, \delta_{\varepsilon}}} \hat{b}(\mathrm{t}, \mathrm{t})\right\rangle \\
& +\int_{\mathbb{R}_{*}^{d}}\left(e^{\left\langle\theta, k\left(\mathrm{t}^{-}, \mathrm{t}^{-}, \mathrm{u}\right)\right\rangle}-1\right) v(\mathrm{du})+\int_{\mathbb{R}_{*}^{d}}\left(e^{\left\{\frac{\delta_{\varepsilon}}{\varepsilon}\left\langle\theta, \mathrm{H}^{\varepsilon, \hat{b}}\left(\mathrm{t}^{-}, \mathrm{t}^{-}, \mathrm{u}\right)\right\rangle\right.}-1\right) v(\mathrm{du}),
\end{aligned}
$$

and where $\widetilde{\mathbb{E}}$ is the associated expectation operator of $\widetilde{\mathbb{P}}$ followed as

$$
\begin{aligned}
& \frac{d \widetilde{\mathbb{P}}}{d \mathbb{P}}:=\exp \left(\frac{\delta_{\varepsilon}}{\varepsilon} \int_{0}^{\left(\sqrt{\varepsilon} / \delta_{\varepsilon}\right)^{2} \mathrm{~T}}\left\langle\theta,\left(\mathrm{I}+\nabla_{y} \hat{\mathrm{b}}+\delta_{\varepsilon} \nabla_{\chi} \hat{\mathrm{b}}\right)^{*} \sigma\right\rangle(\mathrm{s}, \mathrm{s}) \mathrm{d} W_{\mathrm{s}}\right. \\
& \left.-\frac{1}{2}\left(\frac{\delta_{\varepsilon}}{\varepsilon}\right)^{2} \int_{0}^{\left(\sqrt{\varepsilon} / \delta_{\varepsilon}\right)^{2} \mathrm{~T}}\left\langle\theta,\left(\mathrm{I}+\nabla_{y} \hat{\mathrm{b}}+\delta_{\varepsilon} \nabla_{x} \hat{\mathrm{b}}\right)^{*} \sigma\right\rangle^{2}(\mathrm{~s}, \mathrm{~s}) \mathrm{ds}\right) \\
& \times \exp \left(\left(\frac{\delta_{\varepsilon}}{\varepsilon}\right)^{2} \int_{0}^{\left(\sqrt{\varepsilon} / \delta_{\varepsilon}\right)^{2} \mathrm{~T}} \int_{\mathbb{R}_{*}^{\mathrm{d}}}\left\langle\theta, \mathrm{k}\left(\mathrm{s}^{-}, \mathrm{s}^{-}, \mathrm{u}\right)\right\rangle \varepsilon \mathrm{N}^{\varepsilon^{-1}}(\mathrm{duds})\right. \\
& \left.-\left(\frac{\delta_{\varepsilon}}{\varepsilon}\right)^{2} \int_{0}^{\left(\sqrt{\varepsilon} / \delta_{\varepsilon}\right)^{2} T} \int_{\mathbb{R}_{*}^{d}}\left(e^{\left\{\left\langle\theta, k\left(s^{-}, s^{-}, u\right)\right\rangle\right\}}-1\right) v(d u) d s\right) \\
& \times \exp \left(\left(\frac{\delta_{\varepsilon}}{\varepsilon}\right)^{2} \int_{0}^{\left(\sqrt{\varepsilon} / \delta_{\varepsilon}\right)^{2} \mathrm{~T}} \int_{\mathbb{R}_{*}^{\mathrm{d}}}\left\langle\theta, \mathrm{H}^{\varepsilon, \hat{\mathrm{b}}}\left(\mathrm{s}^{-}, \mathrm{s}^{-}, \mathrm{u}\right)\right\rangle \mathrm{N}^{\left(\delta_{\varepsilon} / \varepsilon\right)}(\mathrm{duds})\right. \\
& \left.-\left(\frac{\delta_{\varepsilon}}{\varepsilon}\right)^{2} \int_{0}^{\left(\sqrt{\varepsilon} / \delta_{\varepsilon}\right)^{2} \mathrm{~T}} \int_{\mathbb{R}_{*}^{\mathrm{d}}}\left(e^{\left\{\frac{\delta_{\varepsilon}}{\varepsilon}\left\langle\theta, \mathrm{H}^{\varepsilon, \hat{b}}\left(\mathrm{~s}^{-}, \mathrm{s}^{-}, \mathrm{u}\right)\right\rangle\right\}}-1\right) v(\mathrm{du}) \mathrm{ds}\right) .
\end{aligned}
$$

For all $\theta \in \mathbb{R}^{\mathrm{d}}$, let us set $\Psi^{\varepsilon, \theta} \in \mathrm{C}^{2,1}\left(\mathbb{R}^{\mathrm{d}}, \mathbb{T}^{\mathrm{d}}\right)$ be the unique solution of

$$
\mathcal{L}_{x} \Psi^{\varepsilon, \theta}=\Phi^{\varepsilon, \theta}-\int_{\mathbb{T}^{\mathrm{d}}} \Phi^{\varepsilon, \theta}(x, y) m(x, d y), \quad \text { which satisfies } \int_{\mathbb{T}^{d}} \Psi^{\varepsilon, \theta}(x, y) m(x, d y)=0 .
$$

Such a solution $\Psi^{\varepsilon, \theta}$ must exist again by the assumptions on the coefficients (see for instance, [12, 13]). So we have by applying Itô formula to $\left(\frac{\delta_{\varepsilon}}{\sqrt{\varepsilon}}\right)^{2} \Psi^{\varepsilon, \theta}(\bar{X}, \bar{Y})$ :

$$
\begin{aligned}
& \left(\frac{\delta_{\varepsilon}}{\sqrt{\varepsilon}}\right)^{2} \int_{0}^{\left(\sqrt{\varepsilon} / \delta_{\varepsilon}\right)^{2} t} \Phi^{\varepsilon \theta}(s, s) d s \\
& =\int_{0}^{t} \int_{\mathbb{T}^{\mathrm{d}}} \Phi^{\varepsilon, \theta}\left(X_{s}^{\varepsilon, \delta_{\varepsilon}}, y\right) \mathrm{m}(x, \mathrm{~d} y) \mathrm{ds}+\left(\frac{\delta_{\varepsilon}}{\sqrt{\varepsilon}}\right)^{2}\left[\Psi^{\varepsilon, \theta}\left(\frac{\varepsilon}{\delta_{\varepsilon}^{2}} \mathrm{t}, \frac{\varepsilon}{\delta_{\varepsilon}^{2}} \mathrm{t}\right)-\Psi^{\varepsilon, \theta}\left(x, \frac{x}{\delta_{\varepsilon}}\right)\right] \\
& -\left(\frac{\delta_{\varepsilon}}{\sqrt{\varepsilon}}\right)^{2} \int_{0}^{\left(\sqrt{\varepsilon} / \delta_{\varepsilon}\right)^{2} \mathrm{t}}\left(\delta_{\varepsilon} \nabla_{\chi} \Psi^{\varepsilon, \theta}+\nabla_{y} \Psi^{\varepsilon, \theta}\right) \sigma(s, s) d W_{s} \\
& -\frac{\delta_{\varepsilon}^{3}}{\varepsilon^{2}} \int_{0}^{\left(\sqrt{\varepsilon} / \delta_{\varepsilon}\right)^{2} \mathrm{t}} \nabla_{y} \Psi^{\varepsilon} \mathrm{c}(\mathrm{s}, \mathrm{s}) \mathrm{d} \mathrm{s}+\frac{\delta_{\varepsilon}^{3}}{\varepsilon^{2}} \int_{0}^{\left(\sqrt{\varepsilon} / \delta_{\varepsilon}\right)^{2} \mathrm{t}} \int_{\mathbb{R}_{*}^{d}}\left(\delta_{\varepsilon} \nabla_{\chi} \Psi^{\varepsilon, \theta}+\nabla_{y} \Psi^{\varepsilon, \theta}\right) \mathrm{k}\left(\mathrm{s}^{-}, \mathrm{s}^{-}, z\right) v(\mathrm{~d} z) \mathrm{d} s \\
& -\frac{\delta_{\varepsilon}^{3}}{\varepsilon} \int_{0}^{\left(\sqrt{\varepsilon} / \delta_{\varepsilon}\right)^{2} \mathrm{t}} \operatorname{Tr}\left\{\mathrm{a}\left(\nabla_{\chi} \nabla_{y} \Psi^{\varepsilon, \theta}\right)(\mathrm{s}, \mathrm{s})\right\} \mathrm{d} s-\left(\frac{\delta_{\varepsilon}}{\sqrt{\varepsilon}}\right)^{4} \int_{0}^{\left(\sqrt{\varepsilon} / \delta_{\varepsilon}\right)^{2} \mathrm{t}} \mathcal{L}_{\varepsilon, \delta_{\varepsilon}}^{\bar{\gamma}_{\varepsilon}, \delta_{\varepsilon}} \Psi^{\varepsilon, \theta}(s, s) \mathrm{d} s
\end{aligned}
$$




$$
-\left(\frac{\delta_{\varepsilon}}{\sqrt{\varepsilon}}\right)^{2} \int_{0}^{\left(\sqrt{\varepsilon} / \delta_{\varepsilon}\right)^{2} t} \int_{\mathbb{R}_{*}^{d}} H^{\varepsilon, \Psi^{\varepsilon, \theta}}\left(s^{-}, s^{-}, u\right) N^{\left(\delta_{\varepsilon} / \varepsilon\right)^{2}}(d u d s) .
$$

Then, plugging (3.4) into the formula (3.3) leads to

$$
\begin{aligned}
g_{\mathrm{t}, \mathrm{x}}^{\varepsilon}(\theta)= & \langle x, \theta\rangle+\varepsilon \log \widetilde{\mathbb{E}}\left[\operatorname { e x p } \left\{\frac{1}{\varepsilon} \int_{0}^{\mathrm{t}} \int_{\mathbb{T}^{d}}\left\langle\theta, \Phi^{\varepsilon, \theta}\left(X_{s}^{\varepsilon, \delta_{\varepsilon}}, y\right)\right\rangle \mathrm{m}(x, \mathrm{dy}) \mathrm{ds}-\left(\frac{\delta_{\varepsilon}}{\varepsilon}\right)^{2} \int_{0}^{\left(\sqrt{\varepsilon} / \delta_{\varepsilon}\right)^{2} \mathrm{t}} \mathcal{K}^{\varepsilon, \Psi^{\varepsilon, \theta}}(s, s) \mathrm{d} s\right.\right. \\
& -\left(\frac{\delta_{\varepsilon}}{\varepsilon}\right)^{2} \int_{0}^{\left(\sqrt{\varepsilon} / \delta_{\varepsilon}\right)^{2} \mathrm{t}}\left(\delta_{\varepsilon} \nabla_{x} \Psi^{\varepsilon, \theta}+\nabla_{y} \Psi^{\varepsilon, \theta}\right) \sigma(s, s) \mathrm{d} W_{s} \\
& \left.-\left(\frac{\delta_{\varepsilon}}{\varepsilon}\right)^{2} \int_{0}^{\left(\sqrt{\varepsilon} / \delta_{\varepsilon}\right.}\right)^{2} \mathrm{t} \int_{\mathbb{R}_{*}^{d}} \mathrm{H}^{\varepsilon, \Psi^{\varepsilon, \theta}}\left(s^{-}, \mathrm{s}^{-}, \mathrm{u}\right) \mathrm{N}^{\left(\delta_{\varepsilon} / \varepsilon\right)^{2}}(\mathrm{duds}) \\
& \left.\left.-\frac{\delta_{\varepsilon}}{\varepsilon}\left[\hat{b}\left(\frac{\varepsilon}{\delta_{\varepsilon}^{2}} \mathrm{t}, \frac{\varepsilon}{\delta_{\varepsilon}^{2}} \mathrm{t}\right)-\hat{b}\left(x, \frac{x}{\delta_{\varepsilon}}\right)\right]+\left(\frac{\delta_{\varepsilon}}{\varepsilon}\right)^{2}\left[\Psi^{\varepsilon, \theta}\left(\frac{\varepsilon}{\delta_{\varepsilon}^{2}} \mathrm{t}, \frac{\varepsilon}{\delta_{\varepsilon}^{2}} \mathrm{t}\right)-\Psi^{\varepsilon, \theta}\left(x, \frac{x}{\delta_{\varepsilon}}\right)\right]\right\}\right]
\end{aligned}
$$

where

$$
\begin{aligned}
\mathcal{K}^{\varepsilon, \psi^{\varepsilon, \theta}}(\mathrm{t}, \mathrm{t}):= & \frac{\delta_{\varepsilon}}{\varepsilon} \nabla_{y} \Psi^{\varepsilon, \theta} \mathrm{c}(\mathrm{t}, \mathrm{t})+\delta_{\varepsilon} \operatorname{Tr}\left\{\mathrm{a}\left(\nabla_{\chi} \nabla_{y} \Psi^{\varepsilon, \theta}\right)(\mathrm{t}, \mathrm{t})\right\}+\left(\frac{\delta_{\varepsilon}}{\sqrt{\varepsilon}}\right)^{2} \mathcal{L}_{\mathcal{\varepsilon}, \delta_{\varepsilon}}^{\bar{\gamma}_{\varepsilon}^{\varepsilon, \delta_{\varepsilon}}} \Psi^{\varepsilon, \theta}(\mathrm{t}, \mathrm{t}) \\
& -\frac{\delta_{\varepsilon}}{\varepsilon} \int_{\mathbb{R}_{*}^{\mathrm{d}}}\left(\delta_{\varepsilon} \nabla_{\chi} \Psi^{\varepsilon, \theta}+\nabla_{y} \Psi^{\varepsilon, \theta}\right) \mathrm{k}\left(\mathrm{t}^{-}, \mathrm{t}^{-}, \mathrm{u}\right) v(\mathrm{du}) .
\end{aligned}
$$

First, we have

$$
\begin{aligned}
\left|\log \left[\widetilde{\mathbb{E}}\left(\exp \left\{-\left(\frac{\delta_{\varepsilon}}{\varepsilon}\right)^{2} \int_{0}^{\left(\sqrt{\varepsilon} / \delta_{\varepsilon}\right)^{2} \mathrm{t}}\left(\delta_{\varepsilon} \nabla_{\chi} \Psi^{\varepsilon, \theta}+\nabla_{y} \Psi^{\varepsilon, \theta}\right) \sigma(\mathrm{s}, \mathrm{s}) \mathrm{d} W_{s}\right\}\right)\right]\right| \\
\leqslant \mid \log \left[\widetilde { \mathbb { E } } \left(\exp \left\{-\left(\frac{\delta_{\varepsilon}}{\varepsilon}\right)^{2} \int_{0}^{\left(\sqrt{\varepsilon} / \delta_{\varepsilon}\right)^{2} \mathrm{t}}\left(\delta_{\varepsilon} \nabla_{\chi} \Psi^{\varepsilon, \theta}+\nabla_{y} \Psi^{\varepsilon, \theta}\right) \sigma(\mathrm{s}, \mathrm{s}) \mathrm{d} W_{s}\right\}\right.\right. \\
\quad \times \exp \left\{-\frac{1}{2}\left(\frac{\delta_{\varepsilon}}{\varepsilon}\right)^{4} \int_{0}^{\left(\sqrt{\varepsilon} / \delta_{\varepsilon}\right)^{2} \mathrm{t}}\left\|\left(\delta_{\varepsilon} \nabla_{\chi} \Psi^{\varepsilon, \theta}+\nabla_{y} \Psi^{\varepsilon, \theta}\right) \sigma\right\|^{2}(\mathrm{~s}, \mathrm{~s}) \mathrm{ds}\right\} \\
\left.\left.\quad \times \exp \left\{\frac{1}{2}\left(\frac{\delta_{\varepsilon}}{\varepsilon}\right)^{4} \int_{0}^{\left(\sqrt{\varepsilon} / \delta_{\varepsilon}\right)^{2} \mathrm{t}}\left\|\left(\delta_{\varepsilon} \nabla_{\chi} \Psi^{\varepsilon, \theta}+\nabla_{y} \Psi^{\varepsilon, \theta}\right) \sigma\right\|^{2}(\mathrm{~s}, \mathrm{~s}) \mathrm{ds}\right\}\right)\right] \leqslant \frac{\mathrm{t}}{\varepsilon}\left(\frac{\delta_{\varepsilon}}{\varepsilon}\right)^{2}\left(1+\delta_{\varepsilon}\right)^{2} \mathrm{~K}_{\theta} .
\end{aligned}
$$

Secondly, we can see that:

$$
\begin{aligned}
\mid \log & {\left[\widetilde{\mathbb{E}}\left(\exp \left\{-\left(\frac{\delta_{\varepsilon}}{\varepsilon}\right)^{2} \int_{0}^{\left(\sqrt{\varepsilon} / \delta_{\varepsilon}\right)^{2} \mathrm{t}} \int_{\mathbb{R}_{*}^{\mathrm{d}}} \mathrm{H}^{\varepsilon, \Psi^{\varepsilon, \theta}}\left(\mathrm{s}^{-}, \mathrm{s}^{-}, \mathrm{u}\right) \mathrm{N}^{\left(\delta_{\varepsilon} / \varepsilon\right)^{2}}(\mathrm{duds})\right\}\right)\right] \mid } \\
\leqslant & \mid \log \left[\widetilde { \mathbb { E } } \left(\exp \left\{-\left(\frac{\delta_{\varepsilon}}{\varepsilon}\right)^{2} \int_{0}^{\left(\sqrt{\varepsilon} / \delta_{\varepsilon}\right)^{2} \mathrm{t}} \int_{\mathbb{R}_{*}^{\mathrm{d}}} \mathrm{H}^{\varepsilon, \Psi^{\varepsilon, \theta}}\left(\mathrm{s}^{-}, \mathrm{s}^{-}, \mathrm{u}\right) \mathrm{N}^{\left(\delta_{\varepsilon} / \varepsilon\right)^{2}}(\mathrm{duds})\right\}\right.\right. \\
& \times \exp \left\{-\left(\frac{\delta_{\varepsilon}}{\varepsilon}\right)^{2} \int_{0}^{\left(\sqrt{\varepsilon} / \delta_{\varepsilon}\right)^{2} \mathrm{t}} \int_{\mathbb{R}_{*}^{\mathrm{d}}}\left(e^{\left\{\left(\frac{\delta_{\varepsilon}}{\varepsilon}\right)^{2}\left\langle\theta, \mathrm{H}^{\varepsilon, \Psi^{\varepsilon, \theta}}\left(\mathrm{s}^{-}, \mathrm{s}^{-}, \mathrm{u}\right)\right\rangle\right\}}-1\right) v(\mathrm{du}) \mathrm{ds}\right\} \\
& \left.\left.\times \exp \left\{\left(\frac{\delta_{\varepsilon}}{\varepsilon}\right)^{2} \int_{0}^{\left(\sqrt{\varepsilon} / \delta_{\varepsilon}\right)^{2} \mathrm{t}} \int_{\mathbb{R}_{*}^{\mathrm{d}}}\left(e^{\left\{\left(\frac{\delta_{\varepsilon}}{\varepsilon}\right)^{2}\left\langle\theta, \mathrm{H}^{\varepsilon, \Psi^{\varepsilon, \theta}}\left(\mathrm{s}^{-}, \mathrm{s}^{-}, \mathrm{u}\right)\right\rangle\right\}}-1\right) v(\mathrm{du}) \mathrm{ds}\right\}\right)\right] \leqslant \frac{\mathrm{t}}{\varepsilon}\left(\frac{\delta_{\varepsilon}}{\varepsilon}\right)^{2} \mathrm{~K}_{\theta} .
\end{aligned}
$$


Thirdly, by considering (3.5) one can observe that

$$
\left|\log \left[\widetilde{\mathbb{E}}\left(\exp \left\{-\left(\frac{\delta_{\varepsilon}}{\varepsilon}\right)^{2} \int_{0}^{\left(\sqrt{\varepsilon} / \delta_{\varepsilon}\right)^{2} \mathrm{t}} \mathcal{K}^{\varepsilon, \Psi^{\varepsilon, \theta}}(\mathrm{s}, \mathrm{s}) \mathrm{d} s\right\}\right)\right]\right| \leqslant \frac{\mathrm{t}}{\varepsilon}\left(1+\delta_{\varepsilon}+\frac{\delta_{\varepsilon}}{\varepsilon}\right) \delta_{\varepsilon} \mathrm{K}_{\theta} .
$$

Lastly, a simple computation in terms of solutions to variational problems taking into account the set

$$
\left\{\psi \in \mathcal{D}\left([0, t], \mathbb{R}^{\mathrm{d}}\right): \sup _{0 \leqslant s \leqslant t}\left\|X_{s}^{\varepsilon, \delta_{\varepsilon}}-\varphi_{s}\right\| \leqslant \eta, \varphi_{0}=x\right\}
$$

for $\eta$ small enough, reveals that

$$
\begin{gathered}
\lim _{\varepsilon \rightarrow 0} \varepsilon \log \left[\widetilde{\mathbb{E}}\left(\exp \left\{\frac{1}{\varepsilon} \int_{0}^{t} \int_{\mathbb{T}^{\mathrm{d}}} \Phi^{\varepsilon, \theta}\left(X_{s}^{\varepsilon, \delta_{\varepsilon}}, y\right) m(x, d y) d s\right\}\right)\right] \\
=\inf _{\substack{\varphi \in \mathcal{D}\left([0, t], \mathbb{R}^{\mathrm{d}}\right) \\
\varphi_{0}=x}} \int_{0}^{t} \int_{\mathbb{T}^{\mathrm{d}}} \Phi_{\theta}\left(\varphi_{s}, y\right) m(x, \mathrm{~d} y) \mathrm{d} s
\end{gathered}
$$

where

$$
\begin{aligned}
\Phi_{\theta}:= & \frac{1}{2}\left\langle\theta,\left(\mathrm{I}+\nabla_{y} \hat{b}\right) \sigma\right\rangle^{2}+\left\langle\theta,\left(\mathrm{I}+\nabla_{y} \hat{b}\right)\left(\mathrm{c}-\int_{\mathbb{R}_{*}^{\mathrm{d}}} \mathrm{k}(., ., \mathrm{u}) v(\mathrm{du})\right)\right\rangle \\
& +\int_{\mathbb{R}_{*}^{\mathrm{d}}}\left(e^{\langle\theta, \mathrm{k}(.,, \mathrm{u})\rangle}-1\right) v(\mathrm{du})+\operatorname{Tr}\left\{\mathrm{a}\left(\nabla_{x} \nabla_{y} \hat{\mathrm{b}}\right)\right\} .
\end{aligned}
$$

Altogether, we have shown by considering the estimates (3.6) to (3.7) and the limit (3.8) that

$$
\lim _{\varepsilon \rightarrow 0} g_{t, x}^{\varepsilon}(\theta)=g_{t, x}(\theta)=\Lambda_{1}(\theta)+\Lambda_{2}(\theta),
$$

with

$$
\begin{aligned}
& \Lambda_{1}(\theta):=\inf _{\substack{\varphi \in \mathcal{D}\left([0, t], \mathbb{R}^{\mathrm{d}}\right) \\
\varphi_{0}=\mathrm{x}}}\left\{\frac{1}{2} \int_{0}^{\mathrm{t}} \int_{\mathbb{T}^{\mathrm{d}}}\left\langle\theta,\left(\mathrm{I}+\nabla_{\mathrm{y}} \hat{\mathrm{b}}\right) \sigma\left(\varphi_{s}, y\right)\right\rangle^{2} \mathrm{~m}(\mathrm{x}, \mathrm{dy}) \mathrm{d} s\right. \\
& +\int_{0}^{t} \int_{\mathbb{T}^{d}} \operatorname{Tr}\left\{a\left(\nabla_{x} \nabla_{y} \hat{b}\right)\left(\varphi_{s}, y\right)\right\} m(x, d y) d s+\int_{0}^{t} \int_{\mathbb{T}^{d}}\left\langle\theta,\left(I+\nabla_{y} \hat{b}\right) c\left(\varphi_{s}, y\right)\right\rangle m(x, d y) d s \\
& \left.-\int_{0}^{t} \int_{\mathbb{T}^{\mathrm{d}}}\left\langle\theta,\left(\mathrm{I}+\nabla_{\mathrm{y}} \hat{\mathrm{b}}\right)\left(\varphi_{\mathrm{s}}, \mathrm{y}\right) \int_{\mathbb{R}_{*}^{\mathrm{d}}} k\left(\varphi_{\mathrm{s}^{-}}, \mathrm{y}, \mathrm{u}\right) v(\mathrm{du})\right\rangle \mathrm{m}(\mathrm{x}, \mathrm{d} \mathrm{y}) \mathrm{d} \mathrm{s}\right\}, \\
& \Lambda_{2}(\theta):=\inf _{\substack{\varphi \mathcal{D}\left([0, t], \mathbb{R}^{\mathrm{d}}\right) \\
\varphi_{0}=x}} \int_{0}^{\mathrm{t}} \int_{\mathbb{T}^{\mathrm{d}}} \int_{\mathbb{R}_{*}^{\mathrm{d}}}\left(e^{\left\langle\theta, k\left(\varphi_{s^{-}}, y, u\right)\right\rangle}-1\right) v(\mathrm{du}) \mathrm{m}(x, \mathrm{~d} y) \mathrm{ds} .
\end{aligned}
$$

Clearly $g_{t, x}(\theta)$ is essentially smooth in the sense of hypotheses (i., ii.) of Lemma 2.7, and hypothesis (iii.) of the later lemma holds also true by the requirements on the coefficients.

Now, let us set for $\theta \in \mathbb{R}^{\mathrm{d}}, \overline{\mathrm{X}}_{1}$ be a Wiener process on $\mathbb{R}^{\mathrm{d}}$ with logarithm moment generating function $\Lambda_{1}(\theta)$ (3.9) and $\bar{X}_{2}$ be a Poissonian martingale on $[0, T] \times \mathbb{R}_{*}^{\mathrm{d}}$ independent of $\bar{X}_{1}$ with logarithm moment generating function $\Lambda_{2}(\theta)$ (3.10). 
Let $\bar{J}_{1}(\theta)$ and $\bar{J}_{2}(\theta)$ denote, respectively, the Fenchel-Legendre transform of $\Lambda_{1}(\theta)$ and $\Lambda_{2}(\theta)$, we have

$$
\overline{\mathrm{J}}_{1}(\theta):=\inf _{\substack{\varphi \in \mathcal{D}\left([0, \mathrm{t}], \mathbb{R}^{\mathrm{d}}\right) \\ \varphi_{0}=\mathrm{x}}} \frac{1}{2} \int_{0}^{\mathrm{t}}\left\|\theta-\varphi_{0}-\overline{\mathrm{c}}\left(\varphi_{\mathrm{s}}\right)-\overline{\mathrm{k}}\left(\varphi_{\mathrm{s}^{-}}\right)\right\|_{(\overline{\mathrm{a}})^{-1}\left(\varphi_{\mathrm{s}}\right)}^{2} \mathrm{ds}
$$

and

$$
\overline{\mathrm{J}}_{2}(\theta):=\inf _{\substack{\varphi \in \mathcal{D}\left([0, \mathrm{t}], \mathbb{R}^{\mathrm{d}}\right) \\ \varphi \varphi_{0}=\chi}} \int_{0}^{\mathrm{t}} \int_{\mathbb{T}^{\mathrm{d}}} \int_{\mathbb{R}_{*}^{\mathrm{d}}} \rho\left(\frac{\left\|\theta-\varphi_{0}\right\|}{\left\|\mathrm{k}\left(\varphi_{\mathrm{s}^{-}}, \mathrm{y}, \mathrm{u}\right)\right\|}\right) v(\mathrm{du}) \mathrm{m}(\mathrm{x}, \mathrm{d} \mathrm{y}) \mathrm{ds} .
$$

Note that $g_{t, x}(\theta)$ is the logarithm moment generating of $X_{1}+X_{2}$, hence the Fenchel-Legendre transform of this action functional is $\overline{\mathrm{J}}_{1}(\theta)+\overline{\mathrm{J}}_{2}(\theta)$. For the claim (3.1) of the Theorem 3.1, let $\alpha$ be any element of the sub-differential of $\bar{J}_{1}(\theta)+\bar{J}_{2}(\theta)$ at $\frac{z-x}{t}$, for all $t>0$. Notice that $\bar{J}_{1}$ and $\bar{J}_{2}$ are convex. Then for any $\varphi \in \mathcal{D}\left([0, \mathrm{t}], \mathbb{R}^{\mathrm{d}}\right)$ such that $\varphi_{0}=x$ and $\varphi_{\mathrm{t}}=z$,

$$
\int_{0}^{\mathrm{t}}\left(\overline{\mathrm{J}}_{1}+\overline{\mathrm{J}}_{2}\right)\left(\dot{\varphi}_{\mathrm{s}}\right) \mathrm{ds} \geqslant \mathrm{t}\left(\overline{\mathrm{J}}_{1}+\overline{\mathrm{J}}_{2}\right)\left(\frac{z-x}{\mathrm{t}}\right)+\underbrace{\int_{0}^{\mathrm{t}}\left\langle\alpha, \dot{\varphi}_{\mathrm{s}}-\frac{z-x}{\mathrm{t}}\right\rangle \mathrm{ds}}_{\text {This is zero. }} .
$$

To trace the opposite of (3.11) we have by Jensen's inequality,

$$
\frac{1}{\mathrm{t}} \int_{0}^{\mathrm{t}}\left(\overline{\mathrm{J}}_{1}+\overline{\mathrm{J}}_{2}\right)\left(\dot{\varphi}_{\mathrm{s}}\right) \mathrm{ds} \leqslant\left(\overline{\mathrm{J}}_{1}+\overline{\mathrm{J}}_{2}\right)\left(\frac{1}{\mathrm{t}} \int_{0}^{\mathrm{t}} \dot{\varphi}_{\mathrm{s}} \mathrm{ds}\right)=\left(\overline{\mathrm{J}}_{1}+\overline{\mathrm{J}}_{2}\right)\left(\frac{z-\mathrm{x}}{\mathrm{t}}\right) .
$$

Thus the theorem is verified.

To complete this part, we point out that one can also provide the LDP for $X^{\varepsilon, \delta_{\varepsilon}}$ in pathwise sense. Let us define

$$
S_{0, T}(\varphi):= \begin{cases}\int_{0}^{T}\left(\mathcal{J}_{1}+\mathcal{J}_{2}\right)\left(\dot{\varphi}_{\mathrm{s}}\right) \mathrm{ds}, & \text { if } \varphi \in \mathcal{D}\left([0, \mathrm{~T}], \mathbb{R}^{\mathrm{d}}\right) \text { and } \varphi(0)=x, \\ +\infty, & \text { else. }\end{cases}
$$

Since the functions $\mathcal{J}_{1}$ and $\mathcal{f}_{2}$ are convex we can show that

$$
\inf _{\substack{\varphi \in \mathcal{D}\left([0, T], \mathbb{R}^{\mathrm{d}}\right) \\ \varphi_{0}=x \text { and } \varphi_{\mathrm{T}}=z}} \int_{0}^{\mathrm{T}}\left(\mathcal{J}_{1}+\mathcal{J}_{2}\right)\left(\dot{\varphi}_{\mathrm{s}}\right) \mathrm{ds}:=\mathrm{T}\left(\mathcal{J}_{1}+\mathcal{J}_{2}\right)\left(\frac{z-x}{\mathrm{~T}}\right) .
$$

So we have

Corollary 3.2. Let's set $\mathrm{T}>0$, and assume that the conditions $2.1,2.3$, and 2.4 hold true. Then for every $x \in \mathbb{R}^{\mathrm{d}}$, the family $\left\{X_{\mathrm{T}}^{\varepsilon, \delta_{\varepsilon}}: \varepsilon>0\right\}$ of $\mathcal{D}\left([0, T], \mathbb{R}^{\mathrm{d}}\right)$-valued random variables has an LDP with good rate function $\mathrm{S}_{0, \mathrm{~T}}(\varphi)$ for all $\varphi \in \mathcal{D}\left([0, \mathrm{~T}], \mathbb{R}^{\mathrm{d}}\right)$.

\section{Acknowledgement}

We are grateful to the referees and the guest editors for their constructive critical comments.

\section{References}

[1] D. Applebaum, Léry Processes and Stochastic Calculus, Cambridge University Press, Cambridge, (2004). 1, 2.2

[2] P. Baldi, Large deviations for diffusions processes with homogenization applications, Ann. Probab., 19 (1991), 509-524. 1

[3] P. H. Baxendale, D. W. Stroock, Large deviations and stochastic flows of diffeomorphisms, Probab. Theory Related Fields, 80 (1988), 169-215.

[4] A. Benchérif-Madani, É. Pardoux, Homogenization of a diffusion with locally periodic coefficients, Springer, Berlin, (2005). 2.1 
[5] A. Dembo, O. Zeitouni, Large deviations techniques and applications, Jones and Bartlett Publishers, Boston, (1993). 2.2

[6] P. Dupuis, K. Spiliopoulos, Large deviations for multiscale diffusion via weak convergence methods, Stochastic Process. Appl., 122 (2012), 1947-1987. 1, 1

[7] M. I. Freidlin, R. B. Sowers, A comparison of homogenization and large deviations, with applications to wavefront propagation, Stochastic Process. Appl., 82 (1999), 23-52. 1, 1, 3

[8] N. Ikeda, S. Watanabe, Stochastic differential equations and diffusion processes, North-Holland, New York, (1981). 2.2

[9] H. J. Kushner, Large deviations for two-time-scale diffusions, with delays, Appl. Math. Optim., 62 (2010), 295-322. 1

[10] C. Manga, A. Coulibaly, A. Diedhiou, On Jumps Stochastic Evolution Equations With Application of Homogenization and Large Deviations, J. Math. Res., 11 (2019), 125-134. 1

[11] C. Manga, A. Coulibaly, A. Diedhiou, On some stochastic differential equations with jumps subject to small positives coefficients, AIMS Math., 5 (2019), 1369-1385. 1

[12] E. Pardoux, A. Y. Veretennikov, On the Poisson equation and diffusion approximation. I, Ann. Probab., 29 (2001), 1061-1085. 2.1, 3.2

[13] E. Pardoux, A. Y. Veretennikov, On the Poisson equation and diffusion approximation. II, Ann. Probab., 31 (2003), 1166-1192. 2.1, 3.2

[14] B. Pei, Y. Xu, Mild solutions of local non-Lipschitz stochastic evolution equations with jumps, Appl. Math. Lett., 52 (2016), 80-86. 1

[15] M. Röckner, T. Zhang, Stochastic evolution equations of jump type: existence, uniqueness and large deviation principles, Potential Anal., 26 (2007), 255-279. 1

[16] J. Wu, Uniform large deviations for multivalued stochastic differential equations with Poisson jumps, Kyoto J. Math., 51 (2011), 535-559. 1

[17] Y. Xu, J. Duan, W. Xu, An averaging principle for stochastic dynamical systems with Léry noise, Phys. D, 240 (2011), 1395-1401. 1

[18] Y. Xu, B. Pei, G. Guo, Existence and stability of solutions to non-Lipschitz stochastic differential equations driven by Léry noise, Appl. Math. Comput., 263 (2015), 398-409.

[19] Y. Xu, B. Pei, Y. Li, Approximation properties for solutions to non-Lipschitz stochastic differential equations with Léry noise, Math. Methods Appl. Sci., 38 (2015), 2120-2131. 1

[20] A. Y. Veretennikov, On large deviations for SDEs with small diffusion and averaging, Stochastic Process. Appl., 89 (2000), 69-79. 1

[21] H. Y. Zhao, S. Y. Xu, Freidlin-Wentzell's Large Deviations for Stochastic Evolution Equations with Poisson Jumps, Adv. Pure Math., 6 (2016), 676-694. 1 